\title{
Numerical Simulation of Field Emission in the Surface Conduction Electron-Emitter Display
}

\author{
Hsiang-Yu Lo, Yiming Li, Hsueh-Yung Chao, Chih-Hao Tsai*, Fu-Ming Pan*, \\ Mei-Chao Chiang ${ }^{* *}$, Ting-Chen Kuo ${ }^{* *}$, and Chi-Neng Mo ${ }^{* *}$ \\ Department of Communication Engineering; *Department of Materials Science and \\ Engineering, National Chiao Tung University, Hsinchu 300, Taiwan \\ hylo.cm95g@nctu.edu.tw; ymli@faculty.nctu.edu.tw; hychao@cm.nctu.edu.tw; \\ italno0613@yahoo.com.tw; fmpan@faculty.nctu.edu.tw \\ ${ }^{* *}$ Chunghwa Picture Tubes, Ltd., Taoyuan, Taiwan \\ \{jiangm|kuoku|mocn\}@mail.cptt.com.tw
}

\begin{abstract}
We employ a three-dimensional (3D) particle-in-cell method coupling with finitedifference time domain scheme to simulate the electron emission in surface conduction electron-emitter displays (SEDs). This computational technique includes the space charge effects automatically. We thus explore the conducting mechanism, the emission efficiency, and the current density distribution on the anode plate with one field emission emitter.
\end{abstract}

\section{Introduction}

Surface conduction electron-emitter display (SED) is a new type of flat panel display based on surface conduction electron-emitters (SCEs) [1-3]. The potentialities of SCEs as field emission sources are superior to conventional cathodes in many respects. These SEDs possess high luminance, good color, as well as low power consumption. However, the emission efficiency is determined by the shape and material of SCE. Studies on SCE have not been clearly understood yet. Even if the multiple scattering model of the electron emission mechanism of the SCE has been proposed [4], 3D Maxwell-particle-in-cell simulation [5] has not been considered for the electron emission of SED yet.

\section{Simulation Technique}

The schematic of the emitter structure and related experiment setup is shown in Fig 1(a). The emitter has a coplanar structure with a palladium nanogap which is fabricated by the focused ion beam technology, and the width is approximately $90 \mathrm{~nm}$. Because the dimension of nanogap is much smaller than that of device, a region requires rapid variation in the cell. Therefore, the mesh generation by the Pade function is recommend and the creation of grids near the nanogap region is plotted in Fig. 1(b). We can find that it results in a Pade function-spaced grid over the region. The voltage is $60 \mathrm{~V}$ applied to a conductive film including Pd via a Pt electrode. A 
program has been developed to simulate the emission efficiency and the electron mechanisms of different emission structure. The 3D finite-difference time domain code for self-consistent simulation of the electromagnetic fields and charged particles are performed in our simulation. The computational scheme and the related equations based on physical basis are shown in Fig. 2.

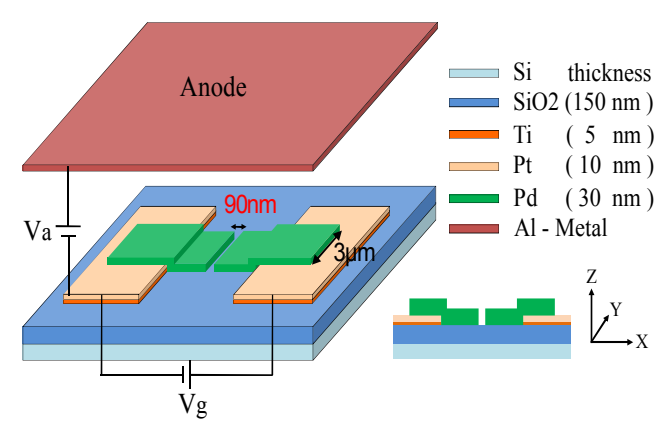

(a)

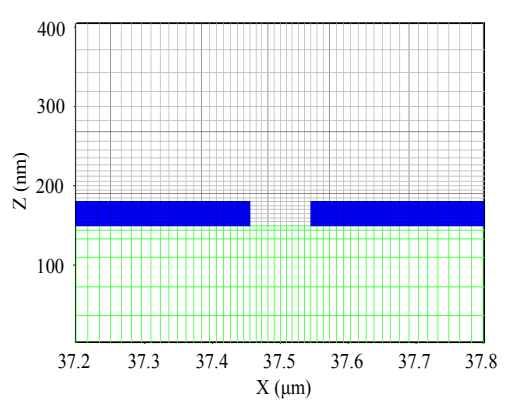

(b)

Figure 1: (a) A schematic plot of the SED structure and the cross section of the surface conduction electron-emitter on the x-z plane. (b) Mesh generation by using the Pade function grids near the nanogap.

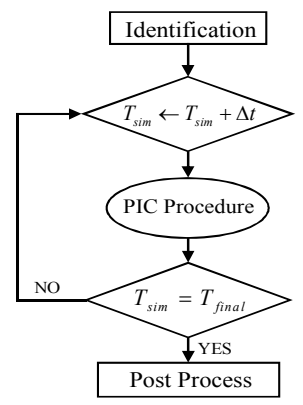

(a)

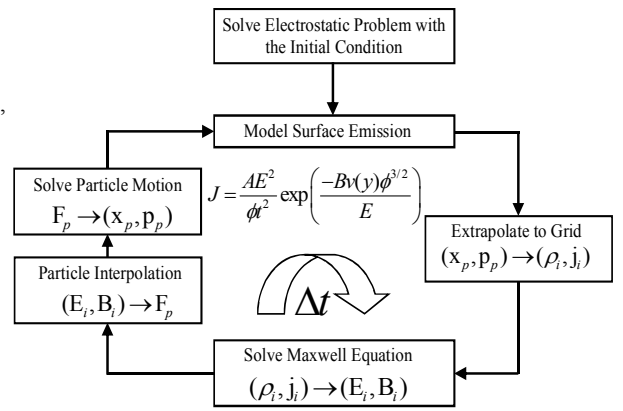

(b)

Figure 2: (a) The computational scheme and the corresponding equations for the electron emission simulation. (b) The flowchart of PIC procedure.

\section{Results and Discussion}

According to our calibrated model [6], high accuracy results could be obtained in this work. Figure 3(a) shows the contour plot of electric fields with the anode voltage (Va) is $3000 \mathrm{~V}$, and the gate voltage $(\mathrm{Vg})$ is $60 \mathrm{~V}$. The fields are higher around the emitter apex and the electrons here are accelerated to energies sufficient to be tunneled from the metal into vacuum. The 3D electron trajectory is shown in Fig. 3(b). The electron beam emits toward the driver electrode and goes upwards to the anode. The cross sections of Fig. 3(b) on the $x-z$ plane with different simulation time are shown in Fig. 4. This is clearer to know the electron-emission mechanism. When the electrons 
emitted to the anode, Fig. 5(a) shows the current density distribution on the anode plate. From this result, the current can be evaluated by integrating the current density to the area. Its value is about $5 \mu \mathrm{A}$ which is large enough to let the phosphor luminance. The light spot which is produced on the phosphor plate of spacer of 500 $\mu \mathrm{m}$ apart from the emitter is shown in Fig. 5(b) when $\mathrm{Va}=3 \mathrm{kV}$ and $\mathrm{Vg}=60 \mathrm{~V}$. The achieved performance is coincided with the suppositions in [1-3].

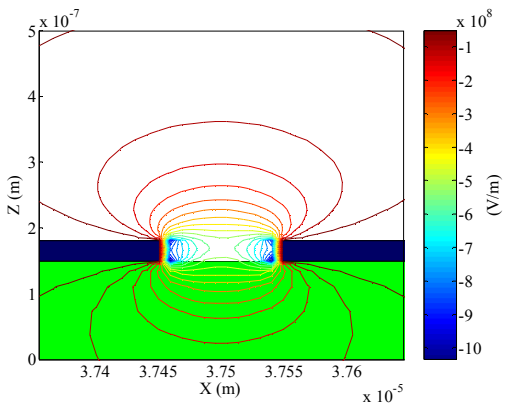

(a)

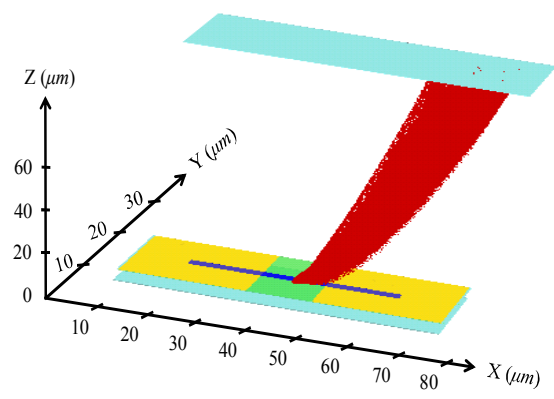

(b)

Figure 3: (a) The contour plot of electric fields in nanogap of $90 \mathrm{~nm}$ wide. (b) The $3 \mathrm{D}$ electron trajectories of the SED structure. The gate voltage is $60 \mathrm{~V}$ and anode voltage is $3000 \mathrm{~V}$, respectively.
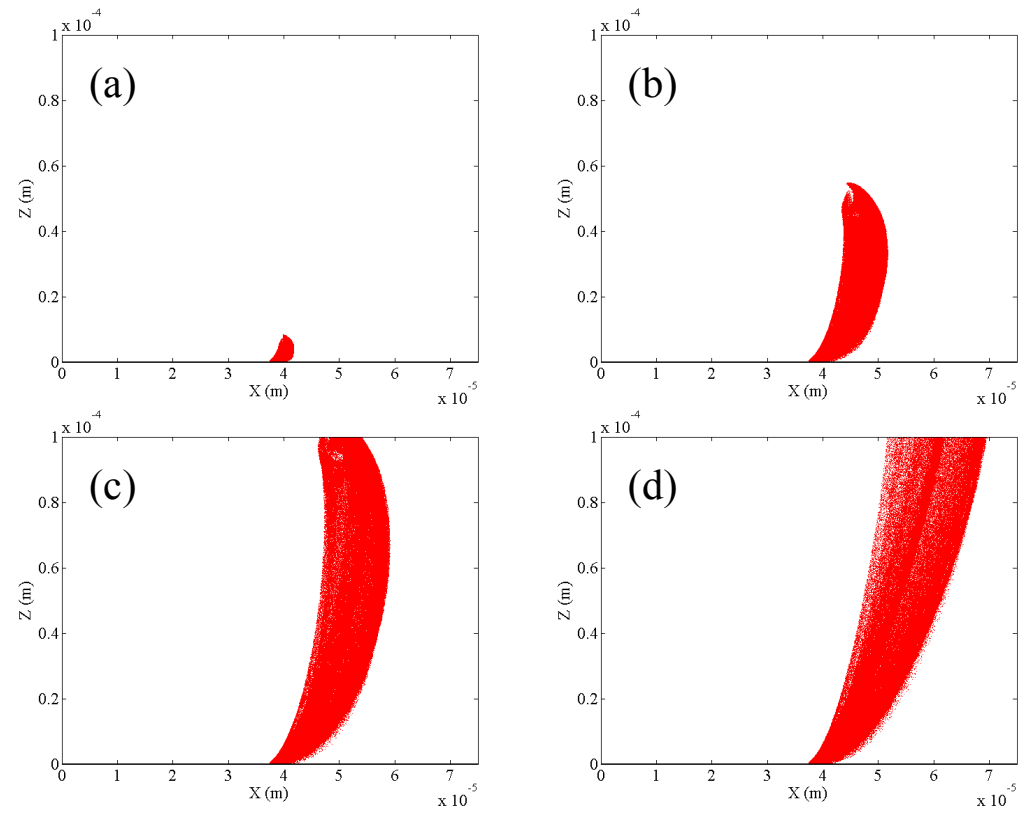

Figure 4: The electron beam ejected from the SCE on the $x-z$ plane with various time. They are $5.6 \mathrm{ps}, 9 \mathrm{ps}, 10 \mathrm{ps}$, and 14 ps from plots (a) to (d), respectively. 


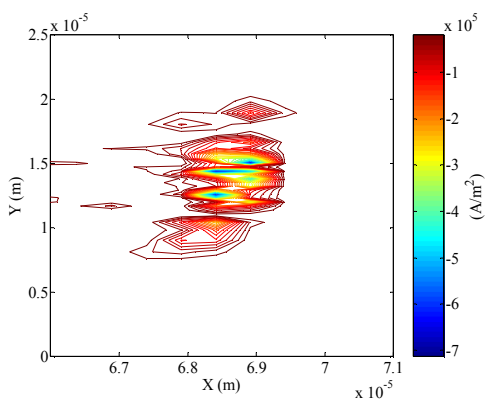

(a)

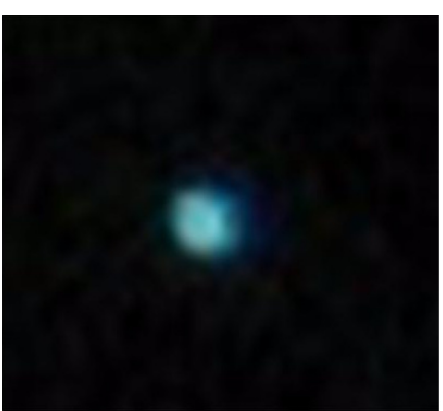

(b)

Figure 5: (a) The simulated current density distribution on the anode plate. (b) The image of a light spot was produced on the phosphor plate.

\section{Conclusions}

We have developed a 3D simulation scheme for the electron emission by using a surface conduction electron emitter. The results including the electron-emission mechanism, current density distribution on the anode plate, and high emission efficiency have been calculated and discussed. They promise the advantages of the SED for TV applications.

\section{Acknowledgements}

This work was supported in part by Taiwan National Science Council (NSC) under Contract NSC-95-2221-E-009-336, Contract NSC-95-2220-E-009-030, Contract NSC-95-2752-E-009-003-PAE, by MoE ATU Program, Taiwan, under a 2006-2007 grant, and by the Chunghwa Picture Tubes under a 2006-2007 grant.

\section{References}

[1] K. Sakai, I. Nomura, E. Yamaguchi, M. Yamanobe, S. Ikeda, T. Hara, K. Hatanaka, Y. Osada, H. Yamamoto, T. Nakagiri, "Flat-Panel Displays Based on Surface-Conduction Electron Emitters," Proc. Euro Display '96, 18.3, 569 (1996).

[2] Asai, M. Okuda, S. Matsutani, K. Shinjo, N. Nakamura, K. Hatanaka, Y. Osada, T. Nakagiri, "Multiple-Scattering Model of Surface-Conduction Electron Emitters," SID '97 DIGEST 10.4, 127 (1997).

[3] E. Yamaguchi, K. Sakai, I. Nomura, T. Ono, M. Yamanobe, N. Abe, T. Hara, K. Hatanaka, Y.Osada, H. Yamamoto, and T. Nakagiri, "A 10-in. Surface-Conduction Electron-Emitter Display,” SID '97 DIGEST, 6.2, 52 (1997).

[4] M. Okuda, S. Matsutani, A. Asai, A. Yamano, K. Hatanaka, T. Hara, T. Nakagirl, "Electron Trajectory Analysis of Surface Conduction Electron Emitter Displays (SEDs)," SID '98 DIGEST, 14.1, 185 (1998).

[5] K. Birdsall and A. B. Langdon, Plasma Physics via Computer Simulation, McGraw-Hill, New York (1985).

[6] H.-Y. Lo, C.-H. Tsai, H.-Y. Chao, Y. Li, F.-M. Pan, T.-C. Kuo, M. Liu, and C.-N. Mo, "Three-Dimensional Simulation of Novel Surface Conduction Electron Emitters," to be presented in Int. SID Symp. Seminar and Exhibition, CA, U.S.A., May 20-25, 2007. 